\title{
Modeling the Economic Costs of Climate Policy: An Overview
}

\author{
Patrik Söderholm \\ Economics Unit, Luleå University of Technology, Luleå, Sweden \\ Email: patrik.soderholm@1tu.se
}

Received February 12, 2012; revised March 6, 2012; accepted March 15, 2012

\begin{abstract}
The overall objective of this paper is to scrutinize previous economic models used to assess the economic costs of climate policy. We pay particular attention to the way in which different model structures and assumptions affect cost estimates, and highlight the limitations and the strengths of different types of modelling approaches. The paper begins by briefly discussing the concept of economic costs, different cost categories (i.e., direct costs, partial equilibrium costs and general equilibrium costs), and the various model approaches that can be used to assess the economic impacts of climate policy (e.g., top-down versus bottom-up models). A systematic review of the main assumptions and methodological choices that underlie different reported cost estimates is presented, and we distinguish between five main types of climate policy cost drivers: a) the baseline scenario; b) the structural characteristics of the models; c) the representation of technological change (e.g., endogenous or exogenous); d) the design of climate policy; and e) the inclusion of non-market costs and benefits. The analysis shows that all these elements help explain model outcomes.
\end{abstract}

Keywords: Economic Costs; Climate Policy; Climate-Economy Models

\section{Introduction}

The balance of evidence suggests that anthropogenic emissions of greenhouse gases-out of which carbon dioxide is the most significant-are having a distinct impact on the global climate [1]. Since the Framework Convention on Climate Change was concluded in 1992, nations have been negotiating commitments to stabilize and then reduce emissions of greenhouse gases, which will otherwise continue to build up in the atmosphere. The debate on climate change policy, particularly with respect to the prospect for achieving a binding international commitment to reduce global emissions, has been heavily focused on the economic costs and feasibility of the proposed mitigation plans.

Some nations, such as the USA and Australia, typically base their decisions to withdraw from, for instance, the Kyoto process in part on the high perceived costs for their respective economies. Also in the countries that have ratified the Kyoto Protocol continued concerns exist, not the least about the future costs of the additional policy measures needed to stabilize greenhouse gas concentrations. This became evident during, for instance, the 2009 Copenhagen (COP15) meeting at which no new global commitment of continued reductions of greenhouse gas (GHG) emissions could be reached.

The assessment of climate policy costs must be based on modelling exercises, which try to combine knowledge based on past experiences (e.g. fuel demand and substitution behaviour) and projections of, for instance, future mitigation options and any associated technical progress. There are many types of models that address the interactions between the energy sectors, the economy and the environment (see Section 3). They share the general advantage that they can be used to ex ante analyse the impact of different policies such as the costs of carbon dioxide mitigation and how the overall burdens are divided across different economic sectors. The policy debate can thus be informed by the model results of the measures of greenhouse gas abatement and their economic consequences, not the least as a basis for setting targets and identifying efficient policy instruments.

Unfortunately, though, scholars tend to disagree, sometimes by quite a wide margin, on the costs of climate policy, e.g., [2-4]. To a large extent the differing estimates can be explained by the general limitations of modelling. All models rely on specific assumptions about the structure of the economy, the policy setting and about important parameters. Given the long-term nature of the climate issue, one of the most serious shortcomings of current models may be the difficulties in avoiding too myopic (or indeed optimistic) assumptions about the future of technological options.

This does not imply, however, that modelling efforts 
are futile. Often they are the only tool we have to address future policy impacts, and we should not (ever) expect any model to alone generate the necessary information to guide policy decisions in the climate field. Instead modelling exercises provide knowledge and insights about, for instance, the main driving factors behind the varying cost estimates as well as about the range and nature of the uncertainties involved. In addition, economic modelling can help to illuminate what types of policy measures are likely to lead to lower rather than higher costs. Such knowledge will-in combination with other informed judgement-provide important inputs into the policy-decision process.

The above suggests that it is useful to take a closer look at the different types of models used to assess climate policy costs. How are cost estimates generated, and to what extent can the different reported cost estimates be explained by the choice of model structure? What are the main advantages and drawbacks of the models used, and what model limitations are the most important to address in cost assessments? Finally, what is the role of climate policy design in determining the costs of climate policy? These are the central questions to be addressed in this paper.

The overall objective of this paper is to critically scrutinize previous economic models used to assess the costs of climate protection. We pay particular attention to the way in which different model structures and assumptions affect cost estimates, and highlight the limitations and the strengths of different types of models and approaches. A scrutiny of these issues can hopefully shed some light on the economic sacrifices that are associated with climate protection, and in turn provide pieces of knowledge that in turn can fuel the current debate on climate policy impacts and help lay people interpret model results and make own judgments. As the climate debate evolves, it is important that stakeholders understand the strengths and weaknesses of economic models of climate policy and use them for at least broad insights, but perhaps not for the specific numbers provided [5].

In any discussion on the cost of climate policy one must be careful in defining what is meant by costs. Costs arise at different levels in the economy, and different types of economic models tend to put emphasis on specific types of costs and - in some cases - neglect other cost categories (see further Sections 2 and 3). However, already at this stage five important general caveats and limitations of the paper are in order. First, it needs to be stressed that costs are relative rather than absolute and total, e.g. [6]. This means that any cost estimate of climate policy action is meaningless if it is not expressed relative to some benchmark or reference scenario (i.e., the no-policy alternative).

Second, this report is solely devoted to cost assess- ments, and does not discuss the benefit side of climate policy. The benefits of climate policy (i.e., emissions reductions) are equivalent to the damage to the climate in the reference scenario that we avoid by undertaking measures to reduce emissions of greenhouse gases. An important implication of this limitation is that one should be careful in referring to climate policy as "cheap" or "expensive" purely on the basis of reported cost estimates. The decision for stringent or weak abatement measures depends not only on the expected cost of abatement, but at least as importantly, on the expected costs of climate change. ${ }^{1}$ The types of analyses discussed in this report represent thus only one side of the climate policy coin.

Third, the paper is primarily concerned with the costs of avoiding/mitigating greenhouse gas emissions following the introduction of climate policy, and does thus not discuss the costs incurred by adapting to actual damages. Stern [8] provides an analysis of the economics of adaptation, and concludes that adaptation strategies will help in dealing with the unavoidable impacts of climate change but there are limits to what they can achieve. Still, an economically efficient climate policy is likely to involve a combination of adaptation and abatement measures.

Fourth, we do not address the problem of how the costs of climate policy are distributed in society. Most cost assessments tend to focus on aggregate impacts, but these impacts are typically not evenly distributed among all members of society (including present and future generations) as well as among different countries. The distribution of costs may determine the political feasibility of stringent climate policies [11], and it is therefore useful to complement aggregate cost assessments with analyses of how these costs are distributed [12]. ${ }^{2}$

A final, fifth, caveat concerns the concept of "climate policy" in itself. Today, with the growing concerns for future climate change, many policy measures have a "climate policy label", but it should be clear that energy and environmental policy instruments also address other policy goals (e.g., security-of-supply concerns, local and regional pollution etc.). The above illustrates that the choice of a relevant system boundary is essential in the cost assessment. Most model studies investigate the cost of the implementation of a carbon price (or an increase in this price). However, this also implies that important

\footnotetext{
${ }^{1}$ The paper shows that the assessment of the costs of climate protection is a complex task, but it is clear that the benefit side of climate policy is probably even more complex to grasp and only poorly understood both empirically and perhaps even theoretically, e.g. [7]. The debate following the publication of the so-called Stern Review illustrates this; the major disagreements concern the analysis of the value of avoided climate change. See Stern [8] as well as the comments from major critics such as, for instance, Nordhaus [9] and Weitzman [10].

${ }^{2}$ Pizer and Kopp [13] provide a survey of different approaches for assessing the distribution of policy costs, and consider the impacts on different households, sectors, regions and generations.
} 
climate policies may already be embedded in the baseline scenario, and these scenarios are often best interpreted as extensions of existing policies rather representing nopolicy scenarios.

The paper proceeds as follows. In the next section we discuss in more detail the concept of economic costs; we present a taxonomy of different costs, and distinguish, in particular, between partial and general equilibrium costs. Non-market costs and the impact of policy design on climate policy costs are also discussed. Section 3 presents the different types of models that can be used to measure these costs, and exemplifies - based on earlier studies - how these models can be used in practice. Different ways of reporting cost estimates are also highlighted and briefly discussed. In Section 4 we review the literature that has attempted to systematically assess why cost estimates tend to differ among different studies and model approaches. A number of factors that affect reported differences across model results are identified and discussed. Finally, Section 5 provides some summarizing remarks and highlights a number of important implications.

\section{Climate Policy Costs: What Do We Mean?}

Economists think of cost differently from financial accountants. While accounting costs involve actual expenses plus depreciation charges for capital equipment, economic costs are the costs of utilizing resources. In this report we are solely concerned with economic costs, and here the concept of opportunity cost is important. An opportunity cost is defined as the value of the alternative foregone by choosing a particular activity. One important implication of this concept is that although no monetary transaction has occurred (and thus no accounting cost is recorded), an activity may nonetheless incur an opportunity cost. For instance, public policy may require (directly or indirectly) that households forego leisure time in order to comply with a new regulation. ${ }^{3}$ In such a case the value of this lost time is an opportunity cost to society. In practice it may be very hard to account for all of these types of costs in the policy assessment [14].

The above also illustrates that in estimating the cost impacts of a policy it is important to define the "boundary" of where costs are to be measured. Cost assessments in the climate policy field must address the fact that even though the policy only targets specific industry sectors, firms or households we generally need to move beyond these entities and also assess how, for instance, the higher costs of a product (now less carbon intensive) will

\footnotetext{
${ }^{3}$ For instance, climate policy instruments (e.g., taxes) may induce households to install new energy-saving equipment. The opportunity cost of this policy involves not only the direct outlays for the equipment but also the own time spent on installing the new equipment or searching for - and negotiating with - a firm who performs the installation.
}

be reflected in the economy as a whole. Hourcade et al. [2] distinguish between four types of costs associated with reducing the emissions of carbon dioxide. These are: a) direct compliance costs; b) partial equilibrium costs; c) general equilibrium costs; and d) non-market costs. In addition to these categories we also discuss the presence of costs related to the existence of inefficient policies. A cost-effective climate policy minimizes the total cost of achieving a given emissions reduction, and for policy purposes it is useful to identify any deviations from this particular allocation of resources. As will be illustrated in Section 4, assumptions about policy design - and any related (in) flexibilities in available compliance strategies - tend to be major drivers of the climate policy cost estimates presented in the literature.

\subsection{Direct Compliance Costs}

Climate policy leads to direct outlays for control, the most intuitive and obvious consequence of the policy. From the perspective of, say, a firm climate policy forces changes in production methods or provides incentives to do so via market-based instruments, and the direct compliance cost equals the change in production costs following these policy-induced alterations. These types of costs may include, for instance, the extra cost of switching from coal to natural gas or the cost of additional energy saving equipment.

Pizer and Kopp [13] discuss a number of methodological problems in properly assessing these types of direct costs. For instance, econometric studies may be appropriate in cases where relevant historical experience in pollution control and input substitution exist, but in many cases the cost analysis must rely on posing questions and/or surveys to engineers active in the pollution abatement field. There exist however a number of limitations with these types of cost surveys, and two problems are particularly worth emphasizing. First, since the engineers approached typically are well-informed only about their specific plant or technology, it can be difficult to extrapolate any information gained to the entire population of plants. Not all firms and households face identical financial costs since, for instance, acquisition, installation and operating costs can vary by location and type of facility. Second, the estimates obtained are typically "out-of-pocket" expenses, and "simply tallying these estimates to estimate total costs ignores many indirect costs and may double-count expenses that are not part of final demand," [13, p. 6]. The indirect costs referred to in this quote are discussed below as components of so-called partial equilibrium costs.

\subsection{Partial Equilibrium Costs}

Partial equilibrium costs include the above direct outlays 
for emission control but also additional indirect burdens on firms and households. These indirect costs may be significant, and include adjustment costs associated with changing production processes. Some examples are paper administration work, the time spent on redesigning production lines to reduce pollution, and reductions in operating flexibility [13]. Policies that induce large capital outlays may also crowd out other productive investments. At the household level, people may also have to forego activities that — as a result of the policy - become more costly. For instance, additional time may need to be allocated to policy compliance, and the household can be forced to use lower quality products, in both cases resulting in lost utility. Switching to an air conditioner that is more energy efficient, may also imply switching to something that is bigger, heavier and uglier. These impacts represent real costs to society, but clearly they may be hard to quantify. A policy also poses indirect costs on government agencies, not the least in the form of activities related to policy enforcement and monitoring.

The above examples are labelled partial equilibrium costs since the consequences of the policy are not being traced through the entire economy. For instance, a partial equilibrium analysis takes into account the fact a price increase on electricity induce consumers and producers of electricity to take action and adjust, but it assumes that other prices in the economy are held constant. In the next sub-section we therefore introduce so-called general equilibrium costs, which take into account the many indirect and feedback effects that can take place within the economy.

\subsection{General Equilibrium Costs}

General equilibrium costs include the indirect impacts that policy targeted towards one market can have on economic decisions in other markets, as well as feedback in the original market, as the economy equilibrates to the new policy. We can illustrate this by considering the case where a higher carbon tax is levied on the carbon content of fossil fuels. The coal industry is one of the sectors that will be directly affected by this tax. When the price of carbon increases, the price of coal (net of tax) will decrease. This will force some coal mines to shut down or at least produce less, but it will also affect negatively, for instance, the firms that provide inputs to the coal industry as well as the labour income from the industry. These latter impacts are not addressed in partial equilibrium analyses, but may still be significant relative to the more obvious impacts measured by the regulated firms, not the least in the case of economy-wide initiatives such as climate policy.

As the economy equilibrates and no distortions - creating deadweight losses - due to taxes or regulations exist elsewhere in the economy, the total cost of a policy can be measured solely on the basis of impacts in the regulated sector. In other words, the marginal cost of an incremental regulation in one specific sector then equals the marginal welfare cost of the same regulation across the entire economy [15]. However, an additional welfare cost to the economy arises when there are existing distortions in other markets. For instance, a tax on carbon dioxide emissions in the energy sector can - by influencing demand and supply in these markets - change the magnitude of existing deadweight losses. That is, the carbon tax not only creates a cost in the energy market where it is imposed, it also influences the cost of existing policies (e.g., a tax on labour income) in other markets. In order to measure the total cost-we need to focus on final demand; otherwise, we run the risk of serious doublecounting. Pizer and Kopp [13] explain this as follows:

"Environmental regulations that raise the price of energy, [...], raise both the cost of energy and the cost of manufactured goods that are enegy-intensive. If we count both the increase in manufacturing costs and the increase in consumer expenditures on higher-priced manufactured goods, we double-count the cost of the regulation." (p. 14)

For this reason it is common practice in modelling analysis to measure costs as the decline in the real gross domestic product (GDP), which comprises final consumption, investment, government spending and net exports. In the new equilibrium, the change in (real) final demand will encompass both the partial equilibrium costs discussed above (passed on to households either in the form of higher prices or as lower factor income), as well as the indirect effects occurring in other markets (see also Section 3.4).

General equilibrium costs can be estimated within so-called computable general equilibrium (CGE) models, which are discussed in more detail in Section 3.2. Such models can be used to consider alternative assumptions about taxes, and address the importance of fiscal responses to environmental policies. The need to maintain government budget constraint as the tax base changes leads also to adjustment in taxes and spending. These adjustments - their size and their characteristics - can have important welfare consequences. In recent decades the potential for using the revenues from environmental taxes to offset tax distortions (e.g., labour taxes) elsewhere in the economy has gained much attention among researchers and politicians. This type of "tax shift" is typically known as revenue recycling and the proposed welfare effects referred to as a double dividend (since the revenues raised in environmental policy both reduce pollution and lower the cost of the tax system) [16].

However, although it is clear that there are environmental benefits following the introduction of an environmental tax, it remains an empirical question whether 
the "second dividend" will emerge in practice compared to the no-policy alternative. This is still a rather controversial issue in the economics literature, and the outcome appears to be dependent, among other things, on the sign of the uncompensated elasticity of labour supply and the pre-existence of involuntary unemployment [17,18]. A weaker form of the "double dividend" is however fairly uncontroversial, namely the case where we compare the tax shift relative to an identical environmental policy but where the revenues are returned as a lump-sum [19].

In the climate policy case this latter result may in fact be just as interesting, given that we are in fact about to implement a carbon tax or emissions trading for carbon dioxide emissions. That is, we may not be choosing between policy and no-policy, but rather between two (or several) ways of returning the revenues from a given policy. For instance, given that the Member States of the European Union have joined the EU emissions trading system (EU ETS) this speaks in favour of auctioning permits rather than providing them for free. ${ }^{4}$

\subsection{Non-Market Costs}

Not all dimensions of human welfare are reflected in the values of goods and services traded in economic markets and the utility that accrues from consuming these; a fraction of the benefits and costs of greenhouse gas control are outside the market. One of the most commonly cited benefits is probably that of avoiding other environmental impacts than those associated with climate change [20, 21]. By discouraging the use of fossil fuels, climate policies will tend to generate reductions of local and regional emissions such as, for instance, sulphur dioxide and nitrogen oxides. These reductions imply in turn fewer damages to human health and the natural environment.

Climate policy may also incur some costs that will not be reflected in the marketplace, such as households' use of leisure time to comply with environmental regulations. Another example is an increase in unemployment (at least over a temporary adjustment period), and the resulting negative psychological costs following this adjustment (apart from the income losses that are already a part of the market mechanisms). Moreover, a central component of climate policy is the substitution of renewable (carbon-free) energy technologies for fossilfuelled technologies. Nevertheless, while an increased reliance on renewable energy avoids carbon dioxide emissions they may also incur costs on society that are not already internalized in the market mechanisms, i.e., external costs associated with local or regional air pol-

\footnotetext{
${ }^{4}$ There is thus an important difference between choosing to implement a market-based environmental policy instrument (rather than do nothing) because it can give rise to 'double dividend' effects, and choosing to lower distortionary taxes (rather than applying lump-sum rebates) given that a market-based policy scheme already has been chosen.
}

lutants. This implies, for instance, that in bottom-up models (see Section 3.1) one should avoid the use of engineering costs, i.e., the case where the cost is solely an attribute of the technology rather than an attribute of the context within which the technology is applied. The former approach will often underestimate total social costs.

\subsection{The Cost of Inefficient Policy Design}

The global nature of the climate change problem complicates the cost analysis in many ways, and has important implications for the costs estimated. Since carbon in the atmosphere gets mixed more or less uniformly, emissions anywhere change concentrations everywhere (although with a long lead time). A cost-effective climate policy requires that the marginal cost of emission reducetion is the same in all places and activities. For this reason different aspects of policy flexibility are of uttermost importance, and a cost-effective policy must allow for both so-called "what" flexibility and "where" flexibility. ${ }^{5}$ "What" flexibility implies that the policy does not mandate certain compliance methods, while "where" flexibility means that the policy permits a broad geographic coverage in the compliance process. These two flexibilities may often be related since some mitigation methods (e.g., substitution of low-carbon fossil fuels for coal) only are available in selected economic sectors and countries. Thus, to the extent that marginal costs vary among sectors, countries and compliance strategies, there exist opportunities for cost savings.

The above implies that any policy that restricts compliance behaviour in terms of ignoring certain compliance methods (e.g., the policy focuses only on carbon dioxide or-even worse- on fossil fuels as an aggregate) will waste resources and raise the costs of that same policy. Economic theory suggests that market-based policy instruments, e.g., those assigning a uniform (based on global warming potentials) price on greenhouse gas emissions, will ensure policy cost-effectiveness. The reason is that all actors will emit up to the point where marginal abatement costs equal this price, and since all actors face the same price the marginal cost of emission reduction will be the same in all activities. Clearly, to the extent that marginal costs vary among regions, there are further opportunities for improved cost-effectiveness through international cooperation.

In sum, the main sources of policy inefficiencies can be thought of as being of two kinds. The first is where climate policy fails to implement a uniform price for greenhouse gas emissions across all sectors and activities of the economy either in the form of a carbon tax or an emissions trading system. This may be the case when

\footnotetext{
${ }^{5}$ In addition, the timing of climate mitigation strategies also affects the cost-effectiveness of climate policy, i.e., so-called "when"-flexibility becomes important.
} 
different policy instruments are applied to different economic sectors and activities (typically implying varying marginal costs of abatement across these activities) unless these can be motivated on, for instance, carbon-leakage grounds, or when some compliance measures are not permitted. The second source of inefficiency is when national climate policy does not make use of the benefits of international cooperation such as emissions trading or joint implementation activities (see further Section 4).

The above illustrates that the concept of economic cost is by no means a straightforward one when applied in practice, and it is important to acknowledge what types of costs are addressed in the analysis. For instance, costs to the firm measured in partial equilibrium analysis ignore general equilibrium changes in output and prices and therefore oversees any welfare changes from altered production levels and distortions in other markets.

This also means that some difficult trade-offs must be resolved in the cost analysis; while general equilibrium analysis may provide a more comprehensive assessment of the economy-wide impacts of a policy it may lack in providing details on the direct compliance costs. Such details may be present in partial equilibrium analyses but at the cost of neglecting the linkages between different sectors of the economy. As we go through a number of specific model approaches in the next chapter, these model differences will become apparent.

\section{Modeling the Costs of Greenhouse Gas Emission Reductions}

In this section we review and comment on the different types of modelling approaches used to assess the economic impacts of climate policy. Traditionally such assessments have rested on the use of two main kinds of models that both address the interactions between energy, economy and environment (E3 models). These are commonly referred to as "top-down" and "bottom-up" models, and they: "differ mainly with respect to emphasis placed on a detailed, technologically based treatment of the energy system [bottom-up], and a theoretically consistent description of the general economy [top-down]," [22, p. 107]. During the recent decades a number of so-called hybrid models have also been developed. These combine features from top-down and bottom-up models; typically they include an energy system component in a top-down model, and therefore add more detail to the energy system compared to the rather aggregate approach found in many top-down models.

\subsection{Bottom-Up Models}

Traditional bottom-up (or systems engineering) models are designed to consider the energy sector in relatively great detail, and they do not include a complete charac- terization of overall economic activity. Grübler et al. [23] assert that " $[\mathrm{b}]$ ottom-up models typically seek to minimize the costs of serving an (often) exogenous energy demand by choosing which technologies to install," (p. 336). These models are optimization models in which the different technological options are specified explicitly, and the total discounted system cost is minimized subject to technological and environmental constraints. Bottomup models can be used to identify, for instance, the leastcost mix of technologies of meeting a given final energy demand for energy services under certain greenhouse gas emissions constraints. The different energy services are either specified in terms of demand curves or derived from an aggregate macroeconomic model. Well-known bottom-up energy models include MARKAL [24], MESSAGE [25], and POLES [26]. All these models employ databases of supply, conversion and end-use technologies, with information on physical inputs and costs. This information can - if aggregated across sectors and technologies - be used to calculate the cost of achieving a given reduction in carbon emissions.

Bottom-up analyses are normally criticized for generating too optimistic results about the prospects for cheap carbon abatement or energy savings, e.g., [2] and [27]. An important reason for this is that they specify technologically feasible "cost-effective" approaches for reducing, for instance, carbon dioxide, but they tend to overlook important barriers to implementation such as management and retraining time and/or capital constraints. The models are thus engineering-oriented and the choice of technology is purely cost-based without much behavioural content. In other words, typical bottom-up models focus on the direct compliance costs but often ignore partial as well as general equilibrium costs. This model limitation is well illustrated in a recent assessment of three studies estimating the cost of the 2020 Californian climate policy goal [28]; the authors conclude that all three assessments systematically underestimate total costs by neglecting important cost components, and by failing to address the effectiveness and the cost of the policy measures required to reduce emissions.

In spite of the above limitations, though, bottom-up models are useful as they can highlight energy inefficiencies and technological opportunities. Often they also serve as a first rough cost assessment, which sometimes may be broadly consistent with estimates of the economy-wide (macroeconomic costs) assessed in top-down studies [8].

\subsection{Top-Down Models}

Top-down models are aggregate models of the entire economy, and they represent the sale of goods and services by producers to households and the reciprocal flow of labour and capital from households to industries. 
Some top-down models also explicitly represent the government, but its role in the flow of goods and production factors may often be passive and designated to collecting taxes and disbursing the revenues to firms and households. In this section we introduce and discuss three types of top-down models used to estimate climate policy costs: a) computable general equilibrium (CGE) models; b) macroeconometric models; and c) neoclassical growth models, including so-called overlapping generation models.

General equilibrium prevails when supply and demand are equalized across all of the inter-connected markets in the economy, and general equilibrium models are in turn "simulations that combine the abstract general equilibrium structure $[\ldots]$ with realistic economic data to solve numerically for the levels of supply, demand and price that support equilibrium across a specified set of markets," [29, p. 1]. The demand and supply conditions in these models are based on assumptions that consumers and producers allocate their resources to maximize their welfare (utility) and profits, respectively. Equilibrium is attained through adjustments in relative prices, which are determined within the model. ${ }^{6}$

CGE models are essentially generalized input-output models in that substitution between different goods and factors of production are allowed. An important advantage of these types of models is therefore that they model the linkages between the different sectors of the economy in an economic-theoretically consistent way. Thus, in a CGE model not only the direct effects of the policy on firms and households are acknowledged, but also the indirect general equilibrium effects. This can be particularly important when analysing the economic impacts of economy-wide policy instruments such as carbon taxes and emissions trading.

The practical use of CGE models involves choosing functional forms and parameters to represent a specific situation with real data, and use this to calculate a numerical solution of the model. Specifying the parameter values of the equations to represent the data (generating a base-year equilibrium observation) is called calibration. For this two types of input data are generally needed. The first type is data describing the initial allocation of resources, and they are usually taken from the National Accounts for a specific year. They include expenditures by production sectors and households on goods and services as well as the division of production factors over producers. The second type of data describes the reactions of the agents to changes in relative prices, as specified in terms of elasticities (e.g., factor substitu-tion elasticities). The impacts of a new policy can be quantified, e.g., as a change in the tax rate. The responses to a policy

\footnotetext{
${ }^{6}$ For an overview of and an introduction to the use of CGE models in the field of environmental economics and climate policy, see, for instance $[29,30]$.
}

change are thus typically derived from previous empirical studies analysing behavioural changes following relative price changes. In other words, in contrast to bottom-up models, traditional CGE estimates of the costs of climate policy are not concerned with exactly what technology is applied but rather what the overall results in terms of, for instance, energy consumption are. Another key parameter is the autonomous energy efficiency index (AEEI), indicating the rate at which price-independent technological change improves energy productivity.

Clearly, high parameter values for AEEI and for energy-related substitution elasticities should normally (ceteris paribus) imply a relatively low cost of greenhouse gas emission reduction. It should be noted however that the choice of specific elasticity estimates is far from a straightforward task. Estimates based on historical data may provide a poor guide to future responses; it is sometimes also claimed that historical data often suggest relatively low values for the economy's flexibility in, for instances, fuel choices [31]. In part this is due to the difficulties in estimating long-run price responses in an ever changing world [32].

During the last two decades a relatively large number of E3 CGE models have been developed. One of the leading E3 models in Europe is the GEM-E3, which permits rather detailed country-level analysis within the European Union (EU), and the cost-effectiveness of the flexible mechanisms of the Kyoto Protocol [33]. Another example is the MS-MRT model, which is a multi-sector, multi-region model that has been used to analyse the global impacts of the Kyoto Protocol with emphasis on the international trade aspects of climate policy (including the effects of international emission permit trade) [34].

Most CGE models are static in the sense that an equilibrium at one point in time is compared to an equilibrium that would emerge when some policy is implemented. $^{7}$ However, over the years several types of dynamic CGE models have been developed. One example is the successor to the OECD GREEN model [35], the MIT EPPA model [36]. The latter is a recursive dynamic multi-regional CGE model of the world economy that has been developed for analysis of climate change policy. It is characterized by a series of individual one-period CGE model simulations and is based on the assumption that firms and households have no forward-looking behaviour. In Viguier et al. [37] the EPPA model is used to investigate the costs of the Kyoto Protocol in the European Union, and to compare the results with those of four other energy-economy models. Another type of dynamic CGE model assumes forward-looking economic agents

\footnotetext{
${ }^{7}$ These CGE approaches are known as comparative static models, and they can thus not be used to investigate the transition path to the new equilibrium.
} 
(often based on the perfect foresight assumption). Jorgensen and Wilcoxen [38] provide one example, and they use econometric estimation of the relevant parameters employing long-term U.S. data.

The critique against CGE models takes different shapes. One weakness of the approach is that it assumes that the economy is at an optimal equilibrium, and any forced change must incur economic costs. This implies that consumers' current choices are always economically efficient. While typical bottom-up models may exaggerate the presence of inefficiencies, CGE models can thus be claimed to underestimate the potential for win-win situations [39]. Moreover, CGE models typically contain sparse information concerning the processes involved in reducing emissions. Most models make simplifying assumptions about emissions reductions technologies and measures: in some models input use of emissions abatement is proportional to overall input use and others assume that abatement involves only capital and labour [40]. It also frequently stressed that the CGE approach is static in assuming that responsiveness to price changes will be the same in the future as in the past, in spite of technological innovation, evolving values and policy measures [31].

Another line of critique concerns the assumptions of perfect competition and constant returns to scale. Combating climate change most likely requires huge changes in the infrastructure and network changes. "An analysis based on neoclassical production functions is misleading in that it ignores the discontinuities, bursts of investment, and uncertainties associated with such changes," [39, p. 11]. Nevertheless, the theoretical consistency of the CGE approach and its ability to address important linkages in the economy as a whole probably explain its current popularity.

The macroeconometric models are-in contrast to the CGE approach - not based on a solid microeconomic foundation. They represent instead (open) demand-driven models, which employ econometric techniques to historical data on consumption prices, incomes, and factor costs to model the final demand for goods and services, and the supply from main sectors such as the energy sector. They can be used to simulate change and/or employed as components in CGE models. Well-known macroeconometric models include the DRI models [41] and E3MG [42].

Models that are based on historical trends, and do not assume firms and households that respond efficiently, can better reflect short-term adjustment costs in respect to climate policy changes, including business cycles, inflation and unemployment. Some macroeconometric models are, however, designed to fit the historical data but also to have long-run properties that are consistent with macroeconomic theory. One example is the so-called
Oxford Global Macroeconomic and Energy Model, which has been used to estimate the GDP impacts of reducing carbon dioxide emissions in various countries [43]. Some of the results from this model indicate that the "combination of macroeconomic rigidities and monetary policy responses to higher energy prices means that the output losses are likely to be substantial in the years immediately following the introduction of a carbon tax [...]," (p. 335). This implies thus that macroeconometric and CGE models can be complementary in assessing short- and long-run responses to climate policy.

The final top-down category of models is the neoclassical growth models, which are based on modern growth theory. These share their micro-economic foundation with CGE models, but (in contrast to the static CGE models) they focus explicitly on the development of the economy over time. The dynamic CGE models are therefore effectively neoclassical growth models. Still, growth models typically assume representative agents and a social welfare function representing household welfare as a function of present and future consumption; they are thus not disaggregated into distinct sectors [44]. The welfare function is discounted over the future, and involves also an aggregate economy-wide production (or cost) function based on inputs of capital, labour and emission-intensive production factors. A famous example is Nordhaus' so-called DICE model [45], while a more recent application is [46].

During the last decade so-called overlapping generations (OLG) models have become more popular. While other growth models assume an infinitely lived consumer, OLG models specify generations that die after some periods and are replaced by new generations (although several generations may also co-exist at any particular point in time). As will be illustrated in Section 4, this modelling choice may have important implications for the assessment of climate policy costs. Dynamic optimization by consumers over long periods of time should, according to theory, improve overall efficiency compared to a more myopic decision-making. Thus, the advent of OLG-models may, ceteris paribus, imply higher policy cost estimates.

\subsection{Hybrid Models}

So-called hybrid models typically combine two or more of the above approaches, but the most common approach is to include an energy systems component (i.e., a bottom-up module) in one of the top-down models [4]. Hence, unlike the traditional top-down models, which employ aggregate production functions, the hybrid models typically distinguish between different energy technologies and explicitly treat the changes in the relative prices between these technologies. They therefore hold promise of being both technology-explicit and behave- 
iourally realistic. ${ }^{8}$ Still, just as the top-down models they assume efficient responses to relative prices changes, implying also that any change brought about by policy will incur economic costs. The bottom-up character of the models means also that they tend to introduce some amount of path dependence in the capital stock, implying that it may be costly to leave the chosen path of fossil-fuelled energy technologies.

A well-known example of a hybrid model is the MERGE model, which has been used extensively to evaluate the regional and global effects of climate policy [48]. It combines a bottom-up representation of the energy supply sector with a general equilibrium formulation of the global economy. ${ }^{9}$ Other examples include the CETA model [49], and the above-mentioned CGE model MS-MRT. Jaccard et al. [31] also develop a hybrid model of the Canadian economy, and illustrates how climate policy cost estimates will depend on whether the analyst takes into account only the direct (financial) costs or also other components of consumer and firm preferences.

\subsection{Measuring and Reporting Cost Estimates}

The costs of climate policy are derived from the above model approaches by comparing simulated outcomes for the economy with and without a specific policy. These costs are normally reported using at least one out of four alternative measures [50]. The first measure is the total direct compliance cost, which equals the marginal cost of the reductions integrated over all emissions reductions. This information can easily be derived from bottom-up models but the direct costs are generally not reported in top-down models. A major weakness of this measure is that it does not address the indirect-general equilibrium-costs. ${ }^{10}$

A second measure is to calculate the carbon price (in terms of a tax or a price on emission permits) required, that is the marginal cost of the last ton of emissions reduced. This measure is straightforward in bottom-up studies but can also be employed in CGE models, although in the latter case this can only be achieved by calculating the implicit marginal reduction costs [51]. This is, as was noted above, because most CGE models make simplifying assumptions about mitigation technology, e.g., input use for mitigation can be assumed to be proportional to overall input use. Still, even if these model specification problems can be overcome, one

\footnotetext{
$\overline{{ }^{8} \text { See Böhringer and Rutherford [47] for a technical discussion on hy- }}$ brid modelling.

${ }^{9}$ It is worth noting that in many hybrid models the level of behavioural detail is quite limited compared to standard CGE models [4].

${ }^{10}$ One benefit of hybrid models is thus that the bottom-up approach can be used to determine the direct costs, while the top-down module can assess the indirect costs [44].
}

weakness of this cost measure is that it is partial in the sense that it only relates to prices rather than to the change in final demand (GDP).

The loss in the gross domestic (or world) product (GDP or GWP) is the third cost measure, and it is widely used. It measures thus the change in final demand, i.e., the total goods and services produced by the economy. Normally this impact is expressed as a percentage change from the reference case (rather than as an absolute measure). ${ }^{11}$ It is worth noting that the correlation between marginal mitigation costs (as expressed by the carbon price) and the GDP impact may not be large [53]. For instance, CGE models "may project lower carbon taxes required to achieve an emissions target, but greater GDP losses than energy sector models if they include a strong negative influence of higher prices on economic growth," [50, p. 34].

Nevertheless, since not all costs are part of GDP this measure does not address all changes in consumer welfare. For instance, in a case where a policy induces an increase in working hours, the welfare cost of lost leisure will not be recorded since GDP does not address chan-ges in non-marketed goods. ${ }^{12}$ For this reason the change in well-being can also be measured in a fourth way, which from an economic-theoretical point of view is the most appealing one. The change in consumer utility can be monetized by computing the so-called equivalent variation $(E V)$, which measures the change in income that would lead to the same change in a consumer's utility as the policy considered. In this way a moneyequivalent of welfare change is generated. One problem, though, is that not all models (in particular the bottom-up ones) can produce equivalent income variation results, something which complicates comparisons.

\subsection{Summarizing Remarks}

Assessing the costs of reducing greenhouse gas emissions is difficult, not the least since many assumptions must be made about how the economy will evolve over a long time without and in the presence of a policy program. Model exercises are required, but it should be clear from the above that no single model can provide all the answers needed. Largely the different model approaches should probably be regarded as complements rather than as substitutes. All model approaches have their weaknesses and their strengths, and for all models consider-

\footnotetext{
${ }^{11}$ Absolute measures can be misleading if quoted out of context, not the least since they depend on the price base chosen. Moreover, if costs are presented in present value terms, an appropriate discount rate must be chosen. Measuring the impacts as percentage changes in GDP avoids these problems and facilitates comparisons across countries and years [52].

${ }^{12}$ Some models may also ignore other ancillary non-market benefits of carbon mitigation, such as the reductions in regional air pollution (see Section 2.4).
} 
able uncertainty exists about both the exogenous factors (e.g., economic growth, technology costs etc.) and the best way to model the behavioural responses. Traditionally, bottom-up models are rich in technical detail, but poor in modelling microeconomic behaviour and macroeconomic feedbacks, while the opposite is true for traditional top-down models.

It is particularly useful to highlight the distinction between the conceptions of the cost of behavioural change in bottom-up and a typical top-down model [54]. In the former case "a technology" represents a particular activity or process, and the costs of climate policy stems from the extra cost of a discrete shift from an activity/process to another. By contrast, in CGE models, for instance, no discrete activities are represented, and instead "technology" is defined by continuous production functions (each specifying the relationship between inputs and outputs). Thus, in the latter case the costs of climate policy are determined by the ease with which the economy can substitute along this production feasibility set.

It should also be noted that the choice between topdown and bottom-up analyses may also be contingent on the types of policies to be analysed. Since, for instance, CGE models "conceives of technological change as an abstract, aggregate phenomenon [...] it only helps policy makers assess top-level policy instruments - such as taxes and tradable permits," [31, p. 55]. However, often policy is frequently concerned with technology-specific policies such as tax credits and subsidies.

\section{Estimates of Climate Policy Costs: Why Do They Differ}

Several previous studies note that the estimated costs of reducing greenhouse gas emissions tend to vary a lot across different models and model runs, e.g., [53,55-57]. The presence of a wide range of cost estimates may undermine policy support for fairly stringent climate policy measures; faced with significant uncertainties of the economic impacts politicians may hesitate whether or not to promote tough measures unless more light can be shed on the true costs of these. This suggests that it is useful to systematically review the main assumptions and methodological choices that underlie previous cost estimates. In this section we first identify and discuss a number of factors that may explain reported differences across various models and model runs. These factors include both those related to policy design assumptions, but, not the least, the different structural characteristics of the models used as well as the ways in which technological change is addressed.

We follow the taxonomy suggested by Fischer and Morgenstern [57], and distinguish between four main types of climate policy cost determinants:
- The baseline (or reference) scenario, which determines the level of ambition in carbon abatement.

- The structural characteristics of the models used. These include, for instance, the representation of substitution possibilities, and geographical and technological detail.

- The representation of technological change, i.e., whether it is assumed to be exogenous or endogenous.

- The design of climate policy, not the least concerning the flexibility permitted in complying with the emission (or stabilization) target.

- The inclusion of non-market costs and benefits, including averted climate damages and side-benefits in the form of reductions in other types of emissions (e.g., sulphur dioxide etc.).

The remainder of this section represents essentially a synthesis of the experiences from other studies and model comparison projects, e.g. $[27,53,56,58]$. It is important to note that during the last decade the research on incorporating endogenous technical change in climate policy models has soared and also its impact on costs are investigated, e.g. $[59,60]$.

\subsection{The Baseline Scenario}

The intent of climate policy is to move the economy into a different emission trajectory, which will meet greenhouse gas reduction targets. Policy cost estimation requires therefore comparison of emissions with and without a specific policy. We thus need a baseline scenario, which describes a counterfactual emissions profile. It should reflect changes over time in, for instance, economic and population growth, the energy intensity of economic output as well as the carbon intensity of energy use [50]. Thus, embedded in the baseline scenario are also input prices, costs, market shares etc. In addition, some projections about (exogenous) technical progress are also needed. This is difficult to achieve but important for the results; technical progress (e.g., higher energy efficiencies) implies that additional GDP growth would result in smaller additional carbon dioxide emissions, compared to previous time periods [61]. Edmonds et al. [62] show the importance of technology assumptions in the baseline on cost estimates. It should be clear that the characteristics of the baseline will affect the cost estimates obtained. A baseline scenario with a high growth in carbon dioxide emissions-e.g., due to pessimistic technology assumptions - implies that all the mitigation scenarios associated with that baseline will require stronger policies to achieve a given emissions reduction target.

However, the structure of the model used, and not the least the assumptions on whether technical progress is assumed to respond to energy prices, also influences the baseline scenario [60]. This implies in turn that a higher 
reference scenario of emissions must not necessarily lead to higher mitigation costs. Fisher and Morgenstern [57] compare models that use marginal abatement costs as the economic impact measure, and note that:

"[...], if marginal abatement costs are low in a particular model because energy technologies are more easily substituted in production and consumption activities then, in the absence of a positive carbon price, input with relatively high carbon content might be used, thereby increasing baseline emissions.” (p. 79)

That is, the emission reduction requirement depends on the baseline, which itself is an output of the model used. This implies that ideally one should not treat required emissions reductions as an independent variable determining abatement costs, although this is typically done in most meta-analyses [27]. The fact that the baseline scenario is a model-output is an important-and sometimes neglected issue also in some bottom-up studies. For instance, Stavins et al. [28] criticize one bottom-up study for assuming that gasoline prices will increase by a certain percentage but neglecting the fact that vehicle purchases and driving habits will be influenced as a result.

It is noteworthy that modellers may often have little special expertise in forecasting baseline emissions and the underlying variables determining emissions. Most model groups and researchers therefore rely on the assumptions from other groups and organization. These include, for instance, the World Bank and the United Nations (for global demographic projections), government reports (for macroeconomic assumptions), and different energy agencies (e.g., EIA) (for assumptions on fuel prices and/or availability of energy resources). Experiences from the USA show that in government estimates of the costs of different environmental regulations it has been more common to overestimate baseline emissions, while the share of underestimations is significantly lower [63]. Still, the same study also shows that over time analysts have gotten better at estimating baseline emissions.

Pielke et al. [64] claim that the standard baseline scenarios of the IPCC are overly optimistic about future energy efficiency improvements and technological progress, and "two thirds or more of all the energy efficiency improvements and decarbonisation of energy supply required to stabilize greenhouse gases is already built into the IPCC reference scenario," (p. 531). This leaves rather small emission reduction targets for explicit climate policies. Their analysis highlights the importance of assessing to what extent spontaneous advances in technological innovation will carry the burden of future emissions reductions on the one hand, and to what extent the weight should be given to explicit policy measures creating the conditions for such innovation to occur. ${ }^{13}$

\subsection{The Structural Characteristics of the Model}

An economic model represents essentially a coherent set of assumptions about the structure and functioning of the economy. It is therefore no surprise that model results will be heavily determined by these same assumptions, which often are simplifications adopted to make the model easier to compute and/or analyse. In this sub-section we present and discuss a number of model assumptions that may influence estimates of the cost of climate policy measures.

Climate policy - in the form of carbon taxes or emissions trading in carbon dioxide-raises the price of fuels in proportion to their carbon content. These changes in relative prices will induce firms and households to seek ways to adapt behaviour in order to avoid this additional burden. Several strategies for substituting away from carbon-intensive activities exist. These include, for instance: a) the substitution of fuels with a low carbon content (e.g., natural gas) for carbon-intensive fuels (e.g., coal); b) the substitution of carbon-free energy sources (e.g., wind power, biomass etc.) for fossil fuels; c) the substitution of other factors of production (e.g., labour and capital) for energy; and d) the substitution of less energy-intensive goods for energy-intensive goods [66].

The easier (i.e., less costly) these substitution strategies are, the lower the overall costs of climate policy. The economic models used to analyse the costs of climate policy differ in the degree to which they represent these substitution possibilities. In CGE models the reaction of the different economic agents to policy changes are specified in terms of price and income elasticities. An important assumption in the case of climate policy is that concerning the size of the elasticity of substitution between different input factors (including energy sources). If the model is highly disaggregated in terms of the number of sectors included (permitting factor and final goods substitution) and the assumed substitution elasticities are high (implying less costly substitution behaviour), we would (ceteris paribus) expect a given policy to imply less burdens on the economy. Some models are instead highly aggregated, neglect-ting major interactions between output and energy use, and overall there appears to be little general agreement on the magnitude (and sometimes even the sign) of the substitution elasticities [56]. In bottom-up studies the substitution possibilities are not related to specific substitution elasticities but

\footnotetext{
${ }^{13}$ Rezai [65] notes that in some climate-economy models (e.g., the DICE model) the baseline scenario is specified in a theoretically inconsistent manner. Specifically, it is assumed that the climate externality is internalized in the sense that the private return does fully include the cost of productive assets on the climate, but mitigation efforts are exogenously constrained to zero.
} 
rather to discrete choices between different technology choices. It is reasonable to envisage that the more abundant these options are, the lower (ceteris paribus) the cost of compliance becomes.

(Dis)aggregation issues are not only related to output sectors and factors of production, but also to the number of geographical regions included in the model. International trade and capital mobility can add to the flexibility in meeting climate targets, not the least in the presence of different flexible mechanisms (e.g., emissions trading and JI activities). Assumptions made about the nature of international linkages therefore become important. For instance, a common modelling strategy is to use a so-called Armington specification, which means that imports are treated as imperfect substitutes for domestically produced goods in determining trade equilibria, e.g. [67] (EPPA) and [68] (GTEM)).

Most models focus on carbon dioxide abatement, thus neglecting the potentially important role that other greenhouse gases (e.g., methane, nitrous oxide) may play. Clearly, allowing for the abatement of these gases - taking into account their relative warming potentials and different lifetimes - may imply lower costs of climate policy since it increases the flexibility in meeting climate stabilization targets. A large majority of the models discussed in this paper do not address other greenhouse gases, but recent modelling efforts address this issue, see in particular [69]. Reily et al. [70] also analysed the relative impact of including or excluding greenhouse gases other than carbon dioxide, and found that both carbon prices and welfare losses were about 33 percent lower when all greenhouse gases were addressed in the model.

The possibilities for substituting away from fossil fuels and other greenhouse gas intensive products depend also on the time frame of the analysis. Substitution behaviour becomes easier as the time for adjustment increases (see more below). Many models do not address the adjustment process, but the outcome is often referred to as a 'long-run' solution. The problem is that it is hard to know when this is attained in practice [39], especially when abatement policies may involve changes in the physical infrastructure.

In a general equilibrium context not only energy-related substitution responses do matter for the assessment of climate policy costs. One important example is the ease with which individuals make trade-offs between consumption and leisure [5]. With climate policy the prices of goods and services, whose production or delivery involves the use of fossil fuels, will rise. As a result people will substitute leisure for consumption (i.e., paid work), and if this substitution effect is significant the consumption loss will be signify-cant as well. A low elasticity of substitution between consumption and leisure would however (ceteris paribus) imply a relatively low eco- nomic impact of the original climate policy.

Another model specification issue concerns the inclusion of a so-called backstop technology. As the price of carbon increases renewable energy technologies become competitive, but various types of models may differ in how they treat the potential and the economic costs of such a shift. A common approach in some models is to assume the existence of a backstop technology, i.e., an alternative energy source available in virtually unlimited quantities at some given price [71]. Examples of backstop technologies used for modelling purposes include advanced solar power, renewable transportation fuels but also future nuclear options such as the plutonium breeder reactor. Some recent models have also included carbon capture and storage as a sort of backstop technology $[72] .{ }^{14}$ Under the assumption of a backstop technology, a carbon free fuel or technology becomes thus perfectly elastic in supply and the price of energy is determined independently of the level of demand [27]. This simple characterization of the backstop concept may also be refined by assuming limited economic availability of the backstop technology, implying increased prices if the increase in use is substantial. ${ }^{15}$ If the backstop technology comes into play and is assumed to generate an unlimited quantity at constant marginal costs, the practical effect is to cap the long-run price of fossil fuels. At the other extreme modellers who exclude backstop technologies, essentially assume that carbon taxes would have to rise indefinitely to keep emissions constant during economic growth. ${ }^{16}$

The impact on the climate policy costs of assumptions concerning backstop technologies is overall ambiguous [57]. It should be clear that if the economy is assumed to rely indefinitely on conventional fuels, the economic impacts of any climate policy could be severe (at least in the absence of technological progress in the fossil fuel sectors). Models assuming that backstop technologies are available at non-increasing prices may therefore predict comparatively low costs but this depends critically on how high these prices are [56].

The above structural characteristics of the climate policy models are more or less explicitly addressed in the specific model formulations applied in practice. Although many of these features are often closely connected to the

\footnotetext{
${ }^{14}$ Still, since the cost of this technology is determined endogenously in the model, it is perhaps best described as a quasi-backstop technology [72]

${ }^{15} \mathrm{This}$ is of course a reasonable assumption for many renewable energy sources such as wind and hydropower. The number of suitable sites is limited, and the most economical sites tend to be used first.

${ }^{16}$ Sue Wing [54] points out that the inclusion of backstop technologies essentially implies assuming semi-endogenous technological change "because the timing of the backstop's penetration is determined by the values of other variables for which the model solved," (p. 552). Still, the modeller must make exogenous assumptions about the cost of the backstop technology and the future date after which the technology becomes available for use.
} 
overall type of model used, it may be relevant to also discuss the possible impact on climate policy costs of the overall model structure. Two separate methodological choices may be of interest here. The first choice concerns the one between top-down and bottom-up models. As was discussed in Section 3, one of the main differences between these model approaches is the level of technological detail; in bottom-up studies specific technologies are essentially modelled at the level of the carbon emitting equipment while in top-down studies the behaviour of the energy sector and the other economic sectors are analysed using aggregate data. This feature is strongly related to the ease of substitution between different production factors and technologies, and for this reason a common hypothesis, e.g., [57], is that models with more "bottom-up" characteristics tend to generate lower costs than traditional top-down models.

An equally important distinction between bottom-up and top-down models concerns the prospects for exploiting any existing efficiencies in energy and factor use. Top-down studies (especially CGE models) suggest that mitigation costs are strictly positive because markets (in the absence of policy intervention) are assumed to operate efficiently. Any policy that impairs this efficiency will be costly [4]. In contrast, results from bottom-up models tend to reveal the existence of substantial efficiency gaps because the energy systems were not optimized, suggesting that a lot of carbon emission could be mitigated at low or even negative costs. The main explanation for the identified efficiency gap in bottom-up models is that these models optimize the energy system, while firms and households do not [73]. It is therefore reasonable to anticipate that bottom-up models will (ceteris paribus) imply lower policy costs than top-down models. It should be noted, though, that in practice it has become harder to make a clear distinction between bottom-up models and top-down models. Most notably, many of the new hybrid models have a top-down CGE treatment of the economy but they also include major bottom-up components.

The second methodological choice, which may have notable impacts on climate policy cost estimates, concerns the selection between different types of top-down models. In the literature, e.g., [27,52,56], most attention has been paid to the use of CGE versus macroeconometric models. From a mitigation-cost perspective it is particularly relevant to note that CGE models can be assumed to reflect long-run responses, thus neglecting any adjustment costs involved in attaining the new equilibrium, while the econometric models build on estimated responses, which tend to capture also short-run costs. ${ }^{17}$

\footnotetext{
${ }^{17}$ For this reason it has been suggested that CGE and macroeconometric models are complementary in assessing short- and long-run responses [74].
}

Thus, in the latter model approach the presence of persistent transitional inefficiencies is acknowledged. We would therefore expect the macroeconometric models to predict higher costs of compliance compared to CGE models, and the more efficiently the economy adapts to the policy the less will the difference between the two approaches become.

As was noted above, the ultimate goal of global climate policy is to stabilize the atmospheric concentration of carbon dioxide and other greenhouse gases. Emissions reductions are thus only to be regarded as means towards this end, and even if emission rates are curbed concentrations could continue to rise for centuries [50]. An important policy question then is how to allocate emissions reduction efforts over time, and we would expect the costs to depend on the time allowed for adjustment. Repetto and Austin [27] develop this line of thinking:

"A target for atmospheric concentrations of $\mathrm{CO}_{2}$ is like a 'carbon budget' that limits total $\mathrm{CO}_{2}$ emissions within a specified period of years. [...] Because the capital stock is durable and because so much equipment and building will ultimately have to be changed, adjustment is a costly process. If the time allowed for transition to a lower emissions path is increased to allow capital stock to be replaced as it wears out, overall abatement costs could be reduced. Also, as research and development yields new superior energy-savings technologies, more low-cost substitutes should be available in later years. Finally, postponing costs reduces them because with a positive return on investment, fewer resources need to be set aside today to meet future costs." (p. 21)

This notion is confirmed in selected earlier studies (e.g., [31] and [75]), and since models differ in the time needed for adjustment this could be a potentially important determinant of the estimated costs of a given climate policy.

A related issue concerns the way in which the time profile of emissions is determined in the models. In some models the existence of long-lived decision makers is assumed; these can optimize the timing of, for instance, abatement efforts over the entire planning period (intertemporal dynamic optimization). Other models, though, instead assume optimization period by period (recursive dynamic). Normally, the former modelling approach should (ceteris paribus) lead to lower costs since it provides more flexibility in choosing the optimal emissions reduction path, e.g. [57,72].

\subsection{The Representation of Technical Change}

It is fair to assert that assumptions about technological change will be key determinants of the long-run costs of climate policy. Technological change is the process by which the economy changes over time in terms of the character of productive activity (e.g., processes used for 
production etc.). Technological progress (advance) enables the production of greater output from the same inputs (or the same output with less input).

Many climate-economy models rely on exogenous representations of technology, i.e., it is assumed that technological changes appear as 'manna from heaven' in the sense that the rate and the direction of technological change are pre-specified in the model and therefore essentially unrelated to market conditions, policies and uncertainties [54]. In practice this may mean that technical improvements are a pre-ordained function of time (unresponsive to policy incentives), or that the model is based on an assumption of autonomous energy efficiency improvement each period applied to energy demand [76]. Still, there exist different approaches to introducing endogenous technological change in climate policy models. This implies "incorporating a feedback mechanism by which policy changes the direction, and possibly the overall level, of [technological change] toward carbonsaving [technological change]," [77, p. 2, emphasis in original].

Following Gillingham et al. [77], it is useful to distinguish between two primary avenues to incorporate endogenous technical change. The first approach is learning-induced technical change in (primarily) bottom-up energy system models. In this representation the unit costs of specific technologies are assumed to be a decreasing function of cumulative experience (typically approximated by cumulative output or capacity) [78]. The majority of bottom-up energy modelling studies indicates that the integration of technical change through learning curves can have dramatic influences on the aggregate costs of climate policy. Results from bottom-up models with zero-carbon technologies and learning-by-doing show low gross costs of mitigation compared to similar models with no learning, and often the reported cost differences are significant compared to a no-learning scenario. ${ }^{18}$ This is also true for hybrid model analyses such as Manne and Richels [80]. Since the cost of energy production becomes cheaper as knowledge and experience build up, this also implies that primary energy use typically is higher in the mitigation scenarios including learning-by-doing compared to similar scenarios excluding such induced technical change.

From an economic efficiency point of view lower mitigation costs at the margin also indicate a greater level of socially optimal emissions mitigation. The net costs of reaching the optimum are therefore likely to be higher than the gross costs (but they may still be relatively low). However, given the uncertainties in defining the optimal

\footnotetext{
${ }^{18}$ For instance, in Barreto and Kypreos [79] the total (discounted) cost reduction due to technological learning is estimated at $40 \%-60 \%$ for the time period 2000-2050, while Manne and Richels [80] report cost reductions of about $40 \%-70 \%$ for the period $2000-2100$ in the case of a maximum carbon concentration of $550 \mathrm{ppmv}$.
}

level of $R \& D$ support it is difficult to determine the socially optimal mitigation level in any case. Nevertheless, this reveals one important weakness of bottom-up energy models. They do not consider the general equilibrium impacts of induced technical change on other sectors of the economy in the sense that redirecting R\&D support to the energy sector has an opportunity cost. There exist studies that do acknowledge this issue, and some of these conclude that the opportunity cost of investment in R\&D may severely raise the gross cost of meeting given carbon policy constraints [81]. The reason is that increased $\mathrm{R} \& \mathrm{D}$ flows into one sector (e.g., renewable energy) imply reduced $R \& D$ - and hence reduced productivity - in the other sectors of the economy. Some recent bottom-up energy models, e.g., [26,82], do consider the allocation of R\&D expenses among competing energy technologies but not among different sectors of the economy.

The second approach to represent endogenous technological change is the R\&D-induced approach, almost exclusively applied in to-down models [54]. This approach lends its theoretical basis largely from the endogenous-growth literature in macroeconomics, e.g., [83], $[84,85]$. The main idea behind this approach is that technological change is the result of investment in R\&D and the ensuing accumulation of a knowledge stock; the quantity of R\&D is determined both by the relative price changes but also by the opportunity cost of R\&D. Thus, in contrast to the learning-by-doing approach resources for R\&D are supplied inelastically, i.e., increased accumulation of knowledge in one sector of the economy will reduce the rate of accumulation in other sectors. Climate policy is thus typically not assumed to increase $R \& D$ in general [54]. One commonly applied approach to the inclusion of endogenous technological change in neoclassical growth models is to add a knowledge stock as an argument in the economy-wide production function. R\&D efforts add to this stock, and thus raise the productivity of output.

An important difference across different R\&D models concerns the extent to which they assume the existence of knowledge spillovers to other firms and economic agents. This may have important impacts on the cost of climate policy. These spillovers compensate for the diminished accumulation of productivity-increasing knowledge in the use of regular factor inputs (capital, labour etc.) that is a result of crowding out by the inducement of carbon-saving $\mathrm{R} \& \mathrm{D}$. The climate policy costs are thus typically amplified if these types of spillovers are ignored.

An important finding in many top-down models (not the least those relying on multi-sector CGE approaches) is that the presence of endogenous technological change leads to lower costs of achieving a given emission reduction target (compared to the case of exogenous techno- 
logical change) [52]. This is however mainly empirical findings, and they can thus not be deduced theoretically. There are namely two aspects of these results that are worth emphasizing. First, in these models, climate policy will induce $R \& D$ and knowledge accumulation in carbon-free energy sectors, but it discourages R\&D in other sectors due to slower growth of output in these latter sectors and due to the (often-assumed inelastic) pool of knowledge-generating resources. This suggests thus that there is a positive and increasing opportunity cost to $\mathrm{R} \& \mathrm{D}$, and this is in sharp contrast to the bottom-up models representing technological change through learning curves. Second, since policy induces R\&D efforts that may have social returns above private returns this generates knowledge spillovers that in turn provide benefits from climate policy above those related to emissions reductions. The impacts on climate policy costs will thus depend largely on whether the models account for knowledge market failures, but also on the explicit parameterization of the R\&D effects. The latter concerns, for instance, the elasticity of supply, or opportunity cost, of additional R\&D, which if inelastic will imply significant crowding-out effects.

\subsection{The Design of Climate Policy}

Reductions in the emissions of carbon dioxide require policy, and we highlight the rather obvious fact that the design of the policy instruments chosen - and their respective reach - can affect the costs for policy compliance. Two issues are discussed here. The first concerns the amount flexibility permitted in attaining the policy goal, while the second issue is about how the revenues from, for instance, carbon taxes and/or permit auctions are used.

As has been discussed above, a cost-effective climate policy requires that the marginal costs of abatement are equalized across different activities, sectors, and regions. In practice this can only be achieved through the use of so-called flexible mechanisms, which allow for both "what" flexibility and "where" flexibility. The Kyoto Protocol outlines three flexible mechanisms: a) Joint Implementation (JI); b) Clean Development Mechanism $(\mathrm{CDM})$; and c) emissions trading. Emission reduction projects implemented in industrialized countries and countries with a transition economy belonging to the same group (the countries listed in Annex I to the Protocol) are configured as JI projects. The emission reductions obtained with these projects are certified as ERUs (Emission Reduction Units). The projects implemented by industrialised countries and countries with a transition economy in the developing countries are classified as CDM projects. The emission reductions generated by these projects are certified as CERs (Certified Emission Reductions). Both ERU and CER certificates correspond to 1 ton $\mathrm{CO}_{2}$ equivalent avoided. These certificates can be used by the country that implements the project to meet the reduction goals subscribed to within the framework of the Protocol. Finally, emissions trading makes it possible to trade (ERU and CER) certificates according to the mechanisms of the free market. As was noted in Section 2 such a policy will be cost-effective since it permits trade to the point where the marginal cost of emission reduction is the same in all places and activities. Policy regimes that restrict the use of any of these mechanisms will therefore imply higher costs for reaching any pre-determined policy goal.

As was noted in Section 2.3, if and how any revenues from climate policy instruments are recycled into the economy is likely to affect the cost of the policy. If one assumes that, say, carbon tax revenues are not fully recycled, the tax will have a deflationary and GDP-reducing impacts on the economy. For this reason economists often assume that revenues are returned in the form of lump-sum rebates (i.e., the amount transferred is completely independent of all taxpayer behaviour); this can be seen as an attempt to separate the economic impacts of the climate policy from those arising from other tax cuts. Yet another alternative is to use the carbon tax revenues (or the revenues from auctioning carbon allowances) to reduce existing taxes that penalize work efforts, savings and investment. The economic costs could then be substantially lower compared to lump-sum revenue recycling due to the distorting nature of the many taxes required for revenue-raising purposes. This suggests, thus, that economic models of climate policy that ignore the potential gains from substituting a carbon tax for a market-distorting tax will generate higher cost estimates than a model that does acknowledge these welfare impacts.

Previous meta-analyses of climate policy cost estimates confirm that the use of the flexible Kyoto mechanisms can be expected to reduce the economic impacts of carbon restrictions. Mechanisms such as emissions trading and JI activities ensure that international abatement efforts can be allocated more efficiently. Moreover, as has been discussed above the carbon taxes assumed to be implemented to reach the set targets yield additional tax revenues. The results from previous meta-studies indicate that models that assume that the revenues collected from the carbon tax will be used to correct economic distortions in other sectors of the economy (rather than returned in the form of lump-sum rebates) generate lower costs. The only exception is the meta-study by Barker et al. [56] who find no statistically significant revenue recycling effect.

\subsection{Averted Climate Change and Air Pollution Damages}

An important model assumption concerns the issue of 
whether the reductions in greenhouse gas emissions are explicitly assumed to avoid some of the economic costs from climate change. Although there are costs of doing nothing, these costs are generally not reflected in the baseline scenarios of most models. Obviously, models that take expected economic damages from climate change into account should predict less severe net economic impacts.

A related story emerges in the case of other air pollutants. By discouraging the use of fossil fuels, climate policy generates a number of side-benefits such as reductions of carbon monoxide, sulphur and nitrogen oxides as well as trace pollutants in exhaust gases. The resulting reductions in damages to health, crops and materials represent real economic benefits, i.e., negative costs. Repetto and Austin [27] argue that the estimated economic savings from reduced air pollution damages can be substantial, and Boyd et al. [86] show that when accounting for such non-climate benefits a reduction of almost 50 percent of baseline emission can be attained at no net economic cost.

\section{Concluding Remarks}

This paper has critically analysed the use of economyenergy-environment models to assess the economic costs of climate policy, with aim of highlighting how the results from different models can be understood and interpreted. We have discussed the economic meaning of costs, and the way in which different types of models-bottom-up and top-down - can generate estimates of the economic costs of policy measures. All model approaches have their weaknesses and their strengths, and overall considerable uncertainty exists about both the exogenous factors and the best way to characterize the behavioural responses. The conception of the cost of behavioural change in bottom-up and top-down models differs; in the former case "a technology" represents a particular activity or process, and the costs of climate policy stems from the extra cost of a discrete shift from an activity/process to another. By contrast, in top-down models no discrete activities are represented, and instead "technology" is defined by continuous production (or cost) functions. This also illustrates the important observation that in bottom-up models emphasis is placed on a representation of technological detail, while top-down models provide a theoretically consistent description of overall economy. This suggests that these two model approaches should be considered complements (rather than substitutes), and they have recently been combined in so-called hybrid models.

The estimated costs of reducing greenhouse gas emissions tend to vary a lot across different models and studies, and in the report we consult previous studies and meta-analyses to systematically review the main assump- tions and methodological choices that underlie different cost estimates. We distinguish between four main types of climate policy cost drivers: a) the baseline scenario; b) the structural characteristics of the models; c) the representation of technological change; d) the design of climate policy; and e) the inclusion of non-market costs and benefits. The analysis shows that all these help explain model outcomes. It is noteworthy that policy design - not the least the degree of flexibility permitted in complying with the emission (or stabilization) target - may significantly affect cost estimates.

It is clear that modelling work in the field of climate policy has its limitation, and there is need for additional research. One of most important limitations of climate policy modelling probably concerns the longer term potential for technological progress and the evolution of new technological pathways. Economic modelling of environmental policy is deemed to be particularly useful "for analysing relatively small changes from a baseline, yet what climate-change models frequently are asked is how an entirely different future might evolve," [87, p. 10]. Nevertheless, this report should not be looked upon as an overall critique of climate policy modelling. On the contrary, modelling can — and should - provide important inputs to the policy process. This requires, though, a thorough understanding of the strengths and the limitations of different model approaches and clear view on how to interpret model results. Climate policy is a learning process, and new model developments have to be sensitive to new policy demands while at the same time improving the models' representation of economic behaviour and policy responses.

\section{Acknowledgements}

The completion of this paper was made possible through financial support from the Swedish Environmental Protection Agency and the Swedish Energy Agency (International Climate Policy Program). Excellent research assistance from Thomas Ejdemo is gratefully acknowledged, as are valuable comments and help from Bengt Johansson, Erika Budh and one anonymous reviewer. Any remaining errors, however, reside solely with the author.

\section{REFERENCES}

[1] S. Solomin, D. Qin, Z. Chen, M. Marquis, K. B. Tignor and H. L. Miller, "Contribution of Working Group I to the Fourth Assessment Report of the IPCC on Climate Change," Climate Change 2007: The Physical Science Basis, Cambridge University Press, Cambridge, 2007, p. 996.

[2] J.-C. Hourcade, K. Halsneas, M. Jaccard, W. D. Montgomery, R. G. Richels, J. Robinson, P. R. Shukla and P. Sturm, "A Review of Mitigation Cost Studies," In: J. P. Bruce, H. Lee and E. F. Haites, Eds., Climate Change, 
1995: Economic and Social Dimensions of Climate Change, Contribution of Working Group III to the Second Assessment Report of the IPCC, University of Cambridge Press, New York, 1996.

[3] T. Barker and P. Ekins, "The Costs of Kyoto for the US Economy," The Energy Journal, Vol. 25, No. 3, 2004, pp. 53-71. doi:10.5547/ISSN0195-6574-EJ-Vol25-No3-4

[4] R. S. J. Tol, "Modelling the Costs of Emission Reduction: Different Approaches," Pacific and Asian Journal of Energy, Vol. 10, No. 1, 2000, pp. 1-7.

[5] J. Peace and J. Weyant, "Insight Not Numbers: The Appropriate Use of Economic Models," Pew Center on Global Climate Change, Arlington, 2008.

[6] C. D. Kolstad and M. Toman, "The Economics of Climate Policy," Resources for the Future, Washington DC, 2000, p. 40.

[7] R. Gerlagh and B. C. C. Van Der Zwaan, "Long-Term Substitutability between Environmental and Man-Made Goods," Journal of Environmental Economics and Management, Vol. 44, No. 2, 2002, pp. 329-345. doi:10.1006/jeem.2001.1205

[8] N. Stern, "The Economics of Climate Change: The Stern Review," Cambridge University Press, Cambridge, 2006.

[9] W. D. Nordhaus, "A Review of the Stern Review on the Economics of Climate Change," Journal of Economic Literature, Vol. 45, No. 3, 2007, pp. 686-702. doi:10.1257/jel.45.3.686

[10] M. L. Weitzman, "A Review of the Stern Review on the Economics of Climate Change," Journal of Economic Literature, Vol. 45, No. 3, 2007, pp. 703-724. doi:10.1257/jel.45.3.703

[11] M. Olson, "The Logic of Collective Action: Public Goods and the Theory of Groups," Harvard University Press, Cambridge, 1965.

[12] P. Söderholm, R. Hildingsson, B. Johansson, J. Khan and F. Wilhelmsson, "Governing the Transition to Low-Carbon Futures: A Critical Survey of Energy Scenarios for 2050," Futures, Vol. 43, No. 10, 2011, pp. 1105-1116. doi:10.1016/i.futures.2011.07.009

[13] W. A. Pizer and R. Kopp, "Calculating the Costs of Environmental Regulation," Resources for the Future, Washington DC, 2003, pp. 3-6.

[14] R. Schmalensee, "The Costs of Environmental Protection," In: M. B. Kotowski, Ed., Balancing Economic Growth and Environmental Goods, American Council for Capital Formation, Center for Policy Research, Washington DC, 1994.

[15] A. Mas-Colell, M. D. Whinston and J. R. Green, "Microeconomic Theory," Oxford University Press, New York, 1995.

[16] L. H. Goulder, "Environmental Taxation and the Double Dividend: A Reader's Guide," International Tax and Public Finance, Vol. 2, No. 2, 1995, pp. 157-183. doi:10.1007/BF00877495

[17] A. L. D. Bovenberg and R. A. De Mooij, "Environmental Levies and Distortionary Taxation," American Economic Review, Vol. 84, No. 4, 1994, pp. 1085-1089.
[18] A. L. Bovenberg and F. Van Der Ploeg, "Optimal Taxation, Public Goods and Environmental Policy with Involuntary Unemployment," Journal of Public Economics, Vol. 62, No. 1-2, 1996, pp. 59-83. doi:10.1016/0047-2727(96)01574-5

[19] L. H. Goulder, I. W. H. Parry and D. Burtraw, "RevenueRaising versus Other Approaches to Environmental Protection: The Critical Significance of Preexisting Tax Distortions," RAND Journal of Economics, Vol. 28, No. 4, 1997, pp. 708-731.

[20] P. Ekins, "Rethinking the Costs Related to Global Warming: A Survey of the Issues," Environmental and Resource Economics, Vol. 6, No. 3, 1995, pp. 231-277. doi:10.1007/BF00705981

[21] A. Krook-Riekkola, E. O. Ahlgren and P. Söderholm, "Ancillary Benefits of Climate Policy in a Small Open Economy: The Case of Sweden," Energy Policy, Vol. 39, No. 9, 2011, pp. 4985-4998. doi:10.1016/j.enpol.2011.06.015

[22] A. Löschel, "Technological Change in Economic Models of Environmental Policy: A Survey," Ecological Economics, Vol. 43, No. 2-3, 2002, pp. 105-126. doi:10.1016/S0921-8009(02)00209-4

[23] A. Grübler, N. Nakićenović and W. D. Nordhaus, "Technological Change and the Environment," Resources for the Future, Washington. DC, 2002.

[24] L.G. Fishbone, G. Giesen, H. A. Goldstein and K. J. Stocks, "User's Guide to MARKAL (BNL/KFA Version 2.0): A Multi-Period, Linear-Programming Model for Energy Systems Analysis," Brookhaven National Laboratory, New York, 1983.

[25] S. Messner, "Endogenized Technological Learning in an Energy Systems Model," Journal of Evolutionary Economics, Vol. 7, No. 3, 1997, pp. 291-313.

[26] N. Kouvaritakis, A. Soria C. Thonet and S. Isoard, "Endogenous Learning in World Post-Kyoto Scenarios: Application of the POLES Model under Adaptive Expectations" International Journal of Global Energy Issues, Vol. 14, No. 1-4, 2000, pp. 222-248. doi:10.1504/IJGEI.2000.004419

[27] R. Repetto and D. Austin, "The Costs of Climate Protection: A Guide for the Perplexed," World Resources Institute, Washington DC, 1997.

[28] T. Schatzki, R. Stavins and J. Jaffe, "Too Good to Be True? An Examination of Three Economic Assessments of California Climate Change Policy," Resources for the Future, Washington DC, 2007, pp. 7-12.

[29] I. S. Wing, "Computable General Equilibrium Models and Their Use in Economy-Wide Policy Analysis: Everything You Ever Wanted to Know (But Were Afraid to Ask)," Center for Energy \& Environmental Studies, Boston University, Boston, 2005.

[30] K. Conrad, "Computable General Equilibrium Models for Environmental Economics and Policy Analysis," In: J. C. J. M. Van Den Bergh, Eds., Handbook of Environmental and Resource Economics, Edward Elgar, Cheltenham, 1999.

[31] M. K. Jaccard, J. Nyboer, C. Bataille and B. Sandownik, 
"Modeling the Cost of Climate Policy: Distinguishing between Alternative Cost Definitions and Long-Run Cost Dynamics," The Energy Journal, Vol. 24, No. 1, 2003, pp. 49-73. doi:10.5547/ISSN0195-6574-EJ-Vol24-No1-3

[32] P. Söderholm "The Modelling of Fuel Use in the Power Sector: A Survey of Econometric Analysis," Journal of Energy Literature, Vol. 4, No. 2, 1998, pp. 3-27.

[33] H. Pan, "The Cost Efficiency of Kyoto Flexible Mechanisms: A Top-Down Study with the GEM-E3 World Model," Environmental Modelling \& Software, Vol. 20, No. 11, 2005, pp. 1401-1411. doi:10.1016/j.envsoft.2004.09.020

[34] P. M. Bernstein, W. D. Montgomery, T. F. Rutherford and G.-F. Yang, "Effects of Restrictions on International Permit Trading: The MS-MRT Model," The Energy Journal, Vol. 20, Special Issue, 1999, pp. 221-256.

[35] J.-M. Burniaux, et al., "Green a Multi-Sector, Multi-Region General Equilibrium Model for Quantifying the Costs of Curbing $\mathrm{CO}_{2}$ Emissions: A Technical Manual," OECD Economics Department, Paris, 1992.

[36] M. H. M. Babiker, J. M. Reily, M. Mayer, R. S. Eckaus, I. S. Wing and R.C. Hyman, "The MIT Emissions Prediction and Policy Analysis (EPPA) Model: Revisions, Sensitivities, and Comparisons of Results," MIT Global Change Joint Program, Cambridge, 2001.

[37] L. L. Viguier, M. H. Babiker and J. M. Reily, "The Costs of the Kyoto Protocol in the European Union," Energy Policy, Vol. 31, No. 5, 2003, pp. 459-481. doi:10.1016/S0301-4215(02)00075-7

[38] D. W. Jorgensen and P. J. Wilcoxen, "Reducing US Carbon Emissions: An Econometric General Equilibrium Assessment," Resource and Energy Economics, Vol. 15, No. 1, 1993, pp. 7-25. doi:10.1016/0928-7655(93)90016-N

[39] T. Barker, "Economic Theory and the Transition to Sustainability: A Comparison of General-Equilibrium and Space-Time-Economics Approaches," Tyndall Centre for Climate Change Research, University of Cambridge, Cambridge, 2004.

[40] D. V. Nestor and C. A. Pasurka, "Alternative Specifications for Environmental Control Costs in a General Equilibrium Framework," Economics Letters, Vol. 48, No. 3-4, 1995, pp. 273-280. doi:10.1016/0165-1765(94)00627-E

[41] R. E. Brinner, M. G. Shelby, J. M. Yanchar and A. Christofaro, "Optimizing Tax Strategies to Reduce Green- house Cases without Curtailing Growth," US Environmental Protection Agency, Washington DC, 1992.

[42] T. Barker, P. Haoran, J. Köhler, R. Warren and S. Winne, "Decarbonising the Global Economy with Induced Technological Change: Scenarios to 2100 Using E3MG," The Energy Journal, Vol. 27, Special Issue, 2006, pp. 143160.

[43] A. Cooper, S. Livermore, V. Rossi, J. Walker and A. Wilson, "Economic Implications of Reducing Carbon Emissions: The Oxford Model," The Energy Journal, Vol. 20, Special Issue, 1999, pp. 335-365.

[44] R. B. Dellink, "Modelling the Costs of Environmental Policy: A Dynamic Applied General Equilibrium Assessment," Edward Elgar, Cheltenham, 2005.

[45] W. D. Nordhaus, "Managing the Global Commons," MIT
Press, Cambridge, 1994.

[46] K. E. Rosendahl, "Cost-Effective Environmental Policy: Implications of Induced Technological Change," Journal of Environmental Economics and Management, Vol. 48, No. 3, 2004, pp. 1099-1121. doi:10.1016/j.jeem.2003.12.007

[47] C. Böhringer and T. F. Rutherford "Combining BottomUp and Top-Down," Energy Economics, Vol. 30, No. 2, 2008, pp. 574-596. doi:10.1016/j.eneco.2007.03.004

[48] A. S. Manne and R. G. Richels, "The Kyoto Protocol: A Cost-Effective Strategy for Meeting Environmental Objectives?" The Energy Journal, Vol. 20, Special Issue, 1999, pp. 1-23.

[49] S. C. Peck and T. J. Teisberg, " $\mathrm{CO}_{2}$ Emissions Control Agreements: Incentives for Regional Participation," The Energy Journal, Vol. 20, Special Issue, 1999, pp. $367-$ 390.

[50] J. P. Weyant, "Costs of Reducing Global Carbon Emissions," The Journal of Economic Perspectives, Vol. 7, No. 4, 1993, pp. 27-46.

[51] A. D. Ellerman and A. Decaux, "Analysis of Post-Kyoto $\mathrm{CO}_{2}$ Emissions Trading Using Marginal Abatement Curves," Joint Program on the Science and Policy of Global Change, MIT Press, Cambridge, 1998.

[52] T. Barker, M. S. Qureshi and J. Köhler, "The Costs of Greenhouse Gas Mitigation with Induced Technical Change: A Meta-Analysis of Estimates in the Literature," Cambridge Center for Climate Change Mitigation Research Department of Land Economy, University of Cambridge, Cambridge 2006.

[53] M. Lasky, "The Economic Costs of Reducing Emissions of Greenhouse Gases: A Survey of Economic Models," Technical Paper Series, Congressional Budget Office, Washington DC, 2003.

[54] I. S. Wing, "Representing Induced Technological Change in Models for Climate Policy Analysis," Energy Economics, Vol. 28, No. 5-6, 2006, pp. 539-562. doi:10.1016/j.eneco.2006.05.009

[55] J. P. Weyant and J. N. Hill, "The Costs of the Kyoto Protocol: A Multi-Model Evaluation," The Energy Journal, Special Issue, 1999.

[56] T. Barker, J. Köhler and M. Villena, "The Costs of Greenhouse Gas Abatement: A Meta-Analysis of PostSRES Mitigation Scenarios," Environmental Economics and Policy Studies, Vol. 5, No. 2, 2000, pp. 135-166.

[57] C. Fisher and R. D. Morgenstern, "Carbon Abatement Costs: Why the Wide Range of Estimates?" Resources for the Future, Washington DC, 2005, pp. 3-42.

[58] J. P. Weyant and J. Hill, "The Costs of the Kyoto Protocol: A Multi-Model Evaluation." The Energy Journal, Special Issue, 1999, pp. 7- 44.

[59] O. Edenhofer, C. Kemfert, K. Lessmann, J. Köhler and M. Grubb, "Induced Technological Change: Exploring Implications for the Economics of Atmospheric Stabilization-Synthesis Report from the Innovation Modeling Comparison Project," The Energy Journal Special Issue: Endogenous Technological Change and the Economics of Atmospheric Stabilisation, 2006, pp. 57-108. 
[60] H. Dowlatabadi, "Sensitivity of Climate Change Mitigation Estimates to Assumptions about Technical Change," Energy Economics, Vol. 20, No. 5-6, 1998, pp. 473-493. doi:10.1016/S0140-9883(98)00009-7

[61] A. Golub, A. Markandya and D. Marcellino, "Does the Kyoto Protocol Cost Too Much and Create Unbreakable Barriers for Economic Growth?" Contemporary Economic Policy, Vol. 24, No. 4, 2006, pp. 520-535. doi:10.1093/cep/byl012

[62] J. A. Edmonds, T. Wilson and R. Rosenzweig, "A Global Energy Technology Strategy Project Addressing Climate Change: An Initial Report on International Public-Private Collaboration," Joint Global Change Research Institute, Prince George's, 2000.

[63] W. Harrington, R. D. Morgenstern and P. Nelson, "On the Accuracy of Regulatory Cost Estimates," Journal of Policy Analysis and Management, Vol. 19, No. 2, 2000, pp. 297-322. doi:10.1002/(SICI)1520-6688(200021)19:2<297::AID-P AM7>3.0.CO;2-X

[64] R. Pielke, T. Wigley and C. Green, "Dangerous Assumptions," Nature, Vol. 452, No. 3, 2008, pp. 531-532. doi: $10.1038 / 452531 \mathrm{a}$

[65] A. Rezai, "The Opportunity Cost of Climate Policy: A Question of Reference," The Scandinavian Journal of Economics, Vol. 113, No. 4, 2011, pp. 885-903. doi:10.1111/j.1467-9442.2011.01681.x

[66] W. R. Cline, "The Economics of Global Warming," Institute for International Economics, Washington DC, 1992.

[67] H. D. Jacoby and I. S. Wing, "Adjustment Time, Capital Malleability and Policy Cost," The Energy Journal, Vol. 20, Special Issue, 1999, pp. 73-92.

[68] V. Tulpule, S. Brown, J. Lim, C. Polidano, H. Pant and B. S. Fischer, "The Kyoto Protocol: An Economic Analysis Using GTEM," The Energy Journal, Vol. 20, Special Issue, 1999, pp. 257-285.

[69] F. C. De La Chesnaye and J. P. Weyant, "EMF 21 Multi-Greenhouse Gas Mitigation and Climate Policy," Energy Modeling Forum and The Energy Journal Special Issue, 2006, pp. 1-32.

[70] J. M. Reily, H. D. Jacoby and R. G. Prinn, "Multi-Gas Contributors to Global Climate Change: Climate Impacts and Mitigation Costs of Non- $\mathrm{CO}_{2}$ Gases," Pew Center on Global Climate Change, Arlington, 2003.

[71] W. D. Nordhaus, "The Allocation of Energy Resources," Brookings Papers on Economic Activity, Vol. 1973, No. 3, 1973, pp. 529-576. doi:10.2307/2534202

[72] O. Kuik, L. Brander and R. S. J. Tol, "Marginal Abatement Costs of Greenhouse Gas Emissions: A Meta-Analysis," Energy Policy, Vol. 37, No. 4, 2009, pp. 1395-1403. doi:10.1016/j.enpol.2008.11.040

[73] N. Mabey and J. Nixon, "Are Environmental Taxes a Free Lunch? Issues in Modelling the Macroeconomic Effects of Carbon Taxes?" Energy Economics, Vol. 19, No. 1, 1997, pp. 29-56. doi:10.1016/S0140-9883(96)01006-7

[74] J.-C. Hourcade and J. Robinson, "Mitigating Factors: Assessing the Costs of Reducing GHG Emissions," Energy
Policy, Vol. 24, No. 10-11, 1996, pp. 863-873. doi:10.1016/S0301-4215(96)00071-7

[75] US Energy Information Administration (EIA), "Kyoto Protocol: Impacts of the Kyoto Protocol on U.S. Energy Markets and Economic Activity," Energy Information Administration, Office of Integrated Analysis and Forecasting and US Department of Energy, Washington DC, 1998.

[76] M. Grubb, J. Köhler and D. Anderson, "Induced Technical Change in Energy and Environmental Modelling: Analytical Approaches and Policy Implications," Annual Review of Energy and the Environment, Vol. 27, pp. 271-308. doi:10.1146/annurev.energy.27.122001.083408

[77] K. Gillingham, R. G. Newell and W. A. Pizer, "Modeling Endogenous Technological Change for Climate Policy Analysis," Resources for the Future, Washington. DC, 2007, pp. 7-14.

[78] K. J. Arrow, "The Economic Implications of Learning by Doing," Review of Economic Studies, Vol. 29, No. 3, 1962, pp. 155-173. doi:10.2307/2295952

[79] L. Barreto and S. Kypreos, "A Post-Kyoto Analysis with the ERIS Model Prototype," International Journal of Global Energy Issues, Vol. 14, No. 1-4, 2000, pp. 262-280. doi:10.1504/IJGEI.2000.004428

[80] A. Manne and R. Richels, "The Impact of Learningby-Doing on the Timing and Costs of $\mathrm{CO}_{2}$ Abatement," Energy Economics, Vol. 26, No. 4, 2004, pp. 603-619. doi:10.1016/j.eneco.2004.04.033

[81] L. H. Goulder and S. H. Schneider, "Induced Technological Change and the Attractiveness of $\mathrm{CO}_{2}$ Abatement Policies," Resource and Energy Economics, Vol. 21, No. 3-4, 1999, pp. 211-253. doi:10.1016/S0928-7655(99)00004-4

[82] L. Barreto and S. Kypreos, "Endogenizing R\&D and Market Experience in the 'Bottom-Up' Energy-Systems ERIS Model," Technovation, Vol. 24, No. 8, 2004, pp. 615-629. doi:10.1016/S0166-4972(02)00124-4

[83] R. E. Lucas Jr., "On the Mechanics of Economic Development," Journal of Monetary Economics, Vol. 22, 1988, pp. 3-42.

[84] P. M. Romer, "Endogenous Technological Change," Journal of Political Economy, Vol. 98, No. 5, 1990, pp. S71-S102. doi:10.1086/261725

[85] G. M. Grossman and E. Helpman, "Endogenous Innovation in the Theory of Growth," Journal of Economic Perspectives, Vol. 8, No. 1, 1994, pp. 24-44. doi:10.1257/jep.8.1.23

[86] R. Boyd, K. Krutilla and W. K. Viscusi, "Energy Taxation as a Policy Instrument to Reduce $\mathrm{CO}_{2}$ Emissions: A Net Benefit Analysis," Journal of Environmental Economics and Management, Vol. 29, No. 1, 1995, pp. 1-24. doi:10.1006/jeem.1995.1028

[87] W. A. Pizer and D. Popp, "Endogenizing Technological Change: Matching the Empirical Evidence to Modelling Needs," Resources for the Future, Washington DC, 2007, pp. 7-11. 